\title{
Conformal Tokamak Geometry for Turbulence Computations
}

\author{
Tiago Tamissa Ribeiro and Bruce Scott
}

\begin{abstract}
Turbulence in magnetised plasmas, with particle gyroradii that are small compared to the device size, consists of 2-D dynamically incompressible fluidlike turbulence in planes perpendicular to the magnetic field and of compressible wavelike dynamics parallel to it. A strong anisotropy between the perpendicular and parallel scales of motion results. The natural coordinates are, therefore, those which follow the field lines. The deformational issues which result from the magnetic shear and variation of the distance between the magnetic flux surfaces with position are treated by judicious choices of coordinate representation. We elucidate the methods by which, in turn, the deformation induced by shear and, then, by the shaping is remedied. Both the physical and the computational considerations are treated since grid isotropicity best represents the small scale turbulence and, at the same time, facilitates multigrid solution of the elliptic equations which are part of the overall system. We present the details of the conformal coordinate system and its implementation, together with an example of its calculation for a realistic tokamak equilibrium case.
\end{abstract}

Index Terms-Tokamak, turbulence, simulations, geometry, deformation

\section{INTRODUCTION}

$\mathbf{T}$ URBULENCE is a fundamental problem in magnetically confined plasmas. The physical understanding of the resulting transport properties relies upon large scale direct numerical simulation efforts. Turbulence in a magnetised plasma, with particle gyroradii that are small compared to the device size, consists of 2-D dynamically incompressible turbulent advection due to $E \times B$ drifting flows in the plane perpendicular to the magnetic field and of compressible electromagnetic electron wave dynamics parallel to it. A space scale separation between perpendicular and parallel dynamics results. The natural coordinates are therefore those which follow the background magnetic field lines in a Clebsch representation [1], wherein the magnetic field is given as

$$
\mathbf{B}=\nabla \chi \times \nabla \xi
$$

with $\chi$ denoting any function of the poloidal flux and $\xi$ denoting an anglelike quantity. $\chi$ and $\xi$ become natural choices for perpendicular coordinates which define a field-aligned system in which both $\mathbf{B} \cdot \nabla \chi$ and $\mathbf{B} \cdot \nabla \xi$ vanish. This can be used on either closed or open magnetic flux surfaces. An exactly aligned computational grid is advantageous in that it allows high resolution of the perpendicular spatial dimensions,

T. .T. Ribeiro is with Max-Planck-Institut für Plasmaphysik, EURATOM Association, D-85748 Garching, Germany and with Associação EURATOM/IST, Instituto de Plasmas e Fusão Nuclear - Laboratório Associado, Instituto Superior Técnico, P-1049-001 Lisboa, Portugal

B. Scott is with Max-Planck-Institut für Plasmaphysik, EURATOM Association, D-85748 Garching, Germany where the smallest scales occur, while leaving a coarser mesh for the parallel dimension, populated with larger spatial scales [2], [3], [4], [5].

The continuous radial variation of the helicity of the magnetic field lines, denominated as the magnetic shear, results in severe deformation of the Clebsch coordinate metric, expressed as a secular variation in the off-diagonal elements with the position along the field line. This grid deformation can strongly affect the results as a consequence of the difficulty in representing the details of the nonlinear vorticity dynamics at finite resolution. Restoring local orthogonality by using a different globally-valid field-aligned system which is locally orthogonal on each perpendicular plane, is a helpful countermeasure [6]. We revisit the mathematics of this re-mapping treatment, which in the simplified geometry of circular, highaspect ratio torii solves the problem efficiently. However, we will show that in shaped geometry, with non-circular torii of intermediate aspect ratio, the Clebsch representation is necessarily problematic, as the perpendicular coordinate volume element is typically more slowly varying along a field line than the metric elements themselves. The variation of inter-surface distance with poloidal position due to the magnetic flux expansion then causes the grid cells to deform in a stretch/squeeze way. Turbulence, on the other hand, tends toward isotropicity at small scales for high enough Reynolds number, generally for a neutral fluid [7], or in the perpendicular plane for magnetised plasmas in either 2-D [8] or 3-D [9] models. We describe a solution for this problem in terms of conformal coordinates in the poloidal plane, constructed to yield a slowly varying ratio of the corresponding metric elements with the position along the field line, and barring exceptional cases, is always within a factor of two of unity. Naturally, in doing so, the straight field line property, which is needed for an efficient parallel dynamics representation, is lost. The wavelike parallel responses are still treated with a straight field line coordinate system which can be cast in terms of a Clebsch representation. The ability to map between both coordinate systems using one-to-one and onto shifts in the angles allows the perpendicular and parallel dynamics to interact in a most natural way, providing for maximally efficient computation. A side benefit is that the conformal property enables the simplest and fastest form of multigrid treatments in solving the elliptic field equations that are part of the model.

This work is organised as follows. Section II gives an overview of the definition of straight field line coordinate systems, proving the equivalence of the Clebsch angle definition among different such coordinate systems, and discussing the 
deformation issues bound to such formulation of the coordinates. Section III introduces the concept of the conformal coordinates constructed to keep the deformation low, and shows the map between these and a field aligned system to be used for the wave dynamics. Section IV presents numerical calculations of both coordinate systems for tokamak magnetohydro-dynamic (MHD) equilibria, analysing the resulting grids and their deformation. The section $\mathrm{V}$ provides a summary of the subject presented as well as an outlook. An analytical result is presented in the appendix A to illustrate in a simple way the differences between conformal and straight field line coordinates in light of the grid deformation.

\section{StRAight FIELD LINE GEOMETRY}

\section{A. Magnetic field pitch and the field alignment}

There is an intrinsic relation between the magnetic field pitch and the ability of the coordinates to be cast in a form in which one of them perfectly aligns to the magnetic field. This can only happen if the field lines are straight when represented against the angle coordinates, yielding the field pitch as a function solely of the magnetic flux. To show this we start by introducing a general coordinate system $(\chi, \vartheta, \zeta)$, which is constructed by specifying which properties it should obey. Assuming nested flux surfaces, with $\chi$ as a magnetic flux label and $\vartheta$ and $\zeta$ as angle coordinates, they should fulfil

$$
B^{\chi}=0 \quad B^{\vartheta}=\mathbf{B} \cdot \nabla \vartheta \quad B^{\zeta}=\mathbf{B} \cdot \nabla \zeta \equiv q B^{\vartheta}
$$

with the Jacobian given by

$$
\nabla \chi \times \nabla \vartheta \cdot \nabla \zeta=\frac{1}{\sqrt{g}} .
$$

Here, $q$ denotes the local pitch of the field lines. Using Eqs. (2-3), we express the background tokamak magnetic field as

$$
\begin{aligned}
\mathbf{B} & =B^{\zeta} \sqrt{g} \nabla \chi \times \nabla \vartheta-B^{\vartheta} \sqrt{g} \nabla \chi \times \nabla \zeta \\
& =B^{\vartheta} \sqrt{g} \nabla \chi \times(q \nabla \vartheta-\nabla \zeta)
\end{aligned}
$$

and add and subtract the term $\vartheta B^{\vartheta} \sqrt{g} \nabla \chi \times \nabla q$ to obtain

$$
\mathbf{B}=B^{\vartheta} \sqrt{g} \nabla \chi \times \nabla(q \vartheta-\zeta)-\vartheta B^{\vartheta} \sqrt{g} \nabla \chi \times \nabla q .
$$

The second term vanishes, enabling the expression to be in a Clebsch form, if and only if $q=q(\chi)$ is a flux function, or label. As a flux function, $q$ measures the number of toroidal turns made by a field line in one complete poloidal turn, and is denominated as the magnetic field pitch parameter or safety factor. If $q=q(\chi)$ it is constant on a magnetic flux surface, which implies that the field lines are straight. This can be seen using its definition Eq. (2) and writing the field line equation

$$
\left.\frac{d \vartheta}{\mathbf{B} \cdot \nabla \vartheta}\right|_{\chi}=\left.\frac{d \zeta}{\mathbf{B} \cdot \nabla \zeta}\right|_{\chi}
$$

In this case, introducing the so called field line label angle-like coordinate, also sometimes designated by Clebsch angle [2],

$$
\xi=q \vartheta-\zeta
$$

re-expresses Eq. (5) as

$$
\mathbf{B}=B^{\vartheta} \sqrt{g} \nabla \chi \times \nabla \xi
$$

which is the Clebsch representation of the magnetic field. In $(\chi, \vartheta, \xi)$ coordinates, of all the contravariant components of the magnetic field, only $B^{\vartheta} \neq 0$, which defines $\vartheta$ as the field following coordinate. This is evident when the parallel gradient operator is expressed in this coordinate system

$$
\nabla_{\|} \equiv \frac{\mathbf{B}}{B} \cdot \nabla=\frac{B^{\vartheta}}{B} \frac{\partial}{\partial \vartheta} .
$$

So, only $\vartheta$ is involved in the parallel wave dynamics, which is what allows for a coarser, and hence more computationally efficient, mesh in this direction. Also noteworthy is the fact that the divergence-free and axisymmetry properties of the magnetic field carried out to the generalised coordinates

$$
\nabla \cdot \mathbf{B}=\left.\frac{1}{\sqrt{g}} \frac{\partial}{\partial \vartheta}\left(\sqrt{g} B^{\vartheta}\right)\right|_{\chi \zeta}=0
$$

imply that the quantity $\sqrt{g} B^{\vartheta}$ must be a flux function. This allows to recast Eq. (4) as

$$
\mathbf{B}=\nabla \psi \times \nabla[h(\psi)(q(\psi) \vartheta-\zeta)]
$$

where $\psi$ is the poloidal magnetic flux divided by $-2 \pi$ and $h(\psi)=(\partial \chi / \partial \psi) B^{\vartheta} \sqrt{g}$ is an arbitrary flux function. For a given representation, both $\mathbf{B}$ and $\psi$ must be invariant of the particular choice of generalised angles $\vartheta$ and $\zeta$. This proves that the field line label angle $\xi$ is the same for all straight field line coordinate systems, up to addition or multiplication by a flux function (this means choice of origin and normalisation, respectively; the requirement that $\xi$ be differentiable leads to a requirement that the choice of origin or branch cut be continuous across flux surfaces).

As a last point, it is useful that the choice on which of the straight field line angle-like coordinates is used to describe the parallel dimension is arbitrary. So far, we used the generalised poloidal angle $\vartheta$ for that purpose, but we can also choose to use the generalised toroidal angle $\zeta$ as the field aligned coordinate. It is trivial to show that, if we recast Eq. (4) as

$$
\mathbf{B}=B^{\zeta} \sqrt{g} \nabla \chi \times\left(\nabla \vartheta-\frac{\nabla \zeta}{q}\right)
$$

and define the field line label angle as

$$
\xi=\vartheta-\frac{\zeta}{q}
$$

this yields

$$
\mathbf{B}=B^{\zeta} \sqrt{g} \nabla \chi \times \nabla \xi .
$$

In this case $B^{\zeta} \neq 0$ is the only non-vanishing contravariant component of the magnetic field, such that it is $\zeta$ which follows the wave dynamics

$$
\nabla_{\|} \equiv \frac{\mathbf{B}}{B} \cdot \nabla=\frac{B^{\zeta}}{B} \frac{\partial}{\partial \zeta} .
$$

While the formulation in Eq. (6) is the standard choice for flux tube geometries, in this work we shall use the one of Eqs. (10-13). In general, coordinate systems which have the properties discussed above are designated field aligned or Clebsch representations. 


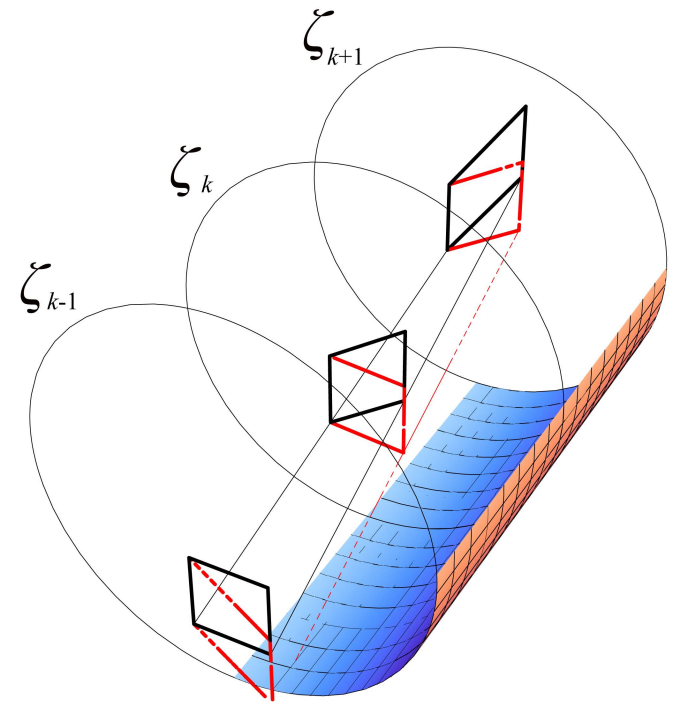

Fig. 1. Illustration of the perpendicular grid-cell deformation along the field lines due to magnetic shear (black). In red, its remedy: the shifted metric procedure to restore locally the perpendicular orthogonality. Here, this is applied at the position $\zeta=\zeta_{k}$

\section{B. Shear deformation}

Independently of the choice for parallel coordinate, Clebsch coordinates possess common characteristics which can be problematic in terms of numerical computations of turbulence. One of such is related to the relation between nonorthogonality and the magnetic shear, which measures the rate of change of the field pitch across flux surfaces. In a tokamak, the latter is finite, such that the non-orthogonality of the perpendicular Clebsch coordinates, measured by the corresponding off-diagonal metric elements reads

$$
\begin{aligned}
g^{\chi \xi} & =\nabla \chi \cdot \nabla \xi=\nabla \chi \cdot \nabla\left(\vartheta-\frac{\zeta}{q}\right) \\
& =g^{\chi \vartheta}-\frac{g^{\chi \zeta}}{q}+\zeta \frac{q^{\prime}}{q^{2}} g^{\chi \chi}
\end{aligned}
$$

The first two terms are a measure of the non-orthogonality of the underlying straight field line coordinates $(\chi, \vartheta, \zeta)$, which typically is not very large. The last term arises from the global magnetic shear since it involves quantity $q^{\prime}=\partial q / \partial \chi$, and it has a secular dependence with the field following (parallel) coordinate, in this case, the generalised toroidal angle $\zeta$. We introduce the following quantity to measure the degree of deformation

$$
d \equiv \frac{g^{\chi \xi}}{g^{\chi \chi}}
$$

whose effect due to the shear is illustrated by the black grid cells along the flux tube depicted in Fig. 1. This secular increase of deformation along the field lines can strongly impact the results, especially in cases where the statistical properties of the vorticity nonlinear dynamics underly the dynamical character [6]. Edge turbulence depends centrally on this physics [9], [10], and the aim is to represent it in the most economical way. The perpendicular laplacian operator is given by

$$
\nabla_{\perp}^{2}=\frac{1}{\sqrt{g}} \frac{\partial}{\partial x^{i}}\left(\sqrt{g} g_{\perp}^{i j} \frac{\partial}{\partial x^{j}}\right)
$$

where the tensor $\mathbf{g}_{\perp}=\mathbf{g}-\mathbf{b b}$ denotes the perpendicular projection. Although the eigenvalues are independent of representation, the additional demand of finite resolution leads to a degraded ability to represent dynamical processes where the metric elements are larger. Hence we desire a representation in which the deformation $d$ is not too large, which means minimisation of the size of the $g^{\chi \xi}$ metric element.

The first step is the use of a different coordinate system on each perpendicular plane (each position along the field lines) where terms involving these processes (including the magnetic drifts) are computed. This is called the shifted metric procedure [6]. It consists of application of a rigid shift (independent of the straight field line angles) to the field line label coordinate $\xi$ so as to yield $g^{\chi \xi}=0$. In its simplest form, the shift does not cancel completely $g^{\chi \xi}$ but rather its global shear dependent part only, given by the last term of Eq. (14). This is illustrated by the red cell in the middle poloidal plane of Fig. 1 corresponding to the position $\zeta=\zeta_{k}$. Such a shift is just $\zeta_{k} / q$, and the new Clebsch angle becomes

$$
\xi_{k}=\vartheta-\frac{\zeta-\zeta_{k}}{q} .
$$

From Fig. 1, it is also clear that to obtain the same effect at a different toroidal (parallel) position, a different shift is to be used. Since different coordinate systems are used at different positions along the field lines, the corresponding shifts need to be taken into account when calculating parallel derivatives, and the branch cut boundary conditions along the field lines are affected (Eqs. 46-50 of Ref. [6]).

\section{Flux expansion deformation}

There is, secondly, an additional cause of strong coordinate cell deformation when the flux surfaced are shaped, inherent to any Clebsch representation. The best way to illustrate it is to express the squared amplitude of the magnetic field in such coordinates, which is inversely proportional to the perpendicular coordinate volume element

$$
\begin{aligned}
B^{2} & =\left(\sqrt{g} B^{\zeta}\right)^{2}\left[g^{\chi \chi} g^{\xi \xi}-\left(g^{\chi \xi}\right)^{2}\right] \\
& \approx\left(\sqrt{g} B^{\zeta}\right)^{2}\left[g^{\chi \chi} g_{k}^{\xi \xi}\right]
\end{aligned}
$$

where the shifted metric procedure described in the previous section was used in the last approximate equality. If one of the contravariant metric elements involved varies faster along the field lines than $B^{2}$, then the remaining one varies inversely. It is convenient to introduce a quantity to measure the ratio of the length of both sides of a perpendicular grid cell, which we denominate by conformal ratio. Using Eq. (17), we can write

$$
R_{c} \equiv \frac{g_{k}^{\xi \xi}}{g^{\chi \chi}} \approx f(\chi) \frac{B^{2}}{\left(g^{\chi \chi}\right)^{2}},
$$

where $f(\chi)=1 /\left(\sqrt{g} B^{\zeta}\right)^{2}$. In the form cast here, we immediately see that this ratio depends strongly on the interspace distance between flux surfaces (measured by $g^{\chi \chi}$ ), the 
so called flux expansion. If this varies considerably along the magnetic field, then the perpendicular grid cells will deform in the stretch/squeeze manner illustrated in Fig. 2. In practice,

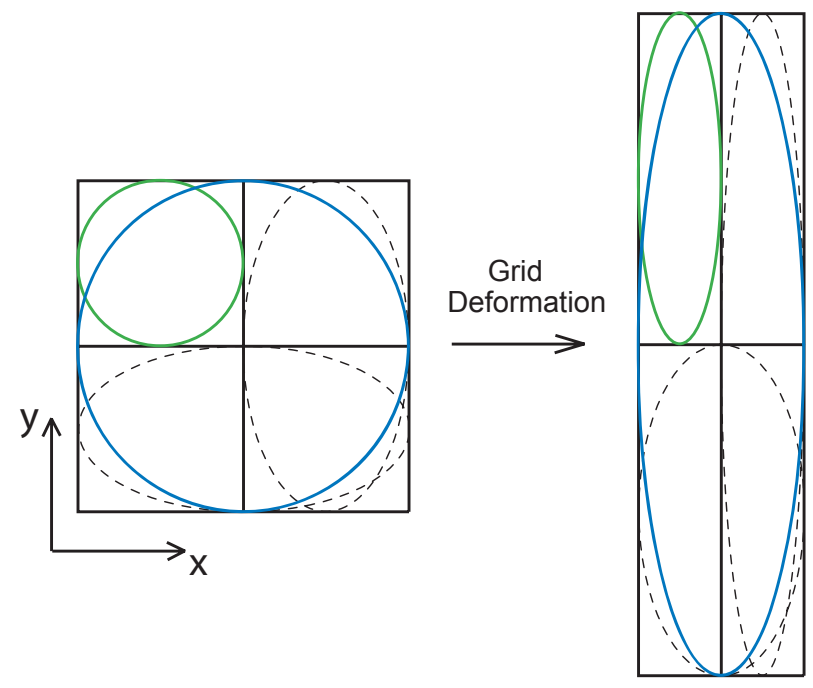

Fig. 2. Illustration of conformal (stretch/compress) deformation of Clebsch coordinates due to magnetic flux expansion. Computationally, the ratio between the metric elements should stay as constant as possible, and that is not the case for strongly shaped geometry.

since the corresponding wavenumbers are

$$
k_{\chi}=\sqrt{g^{\chi \chi}} \frac{2 \pi}{\lambda_{\chi}} \quad k_{\xi}=\sqrt{g_{k}^{\xi \xi}} \frac{2 \pi}{\lambda_{\xi}}
$$

this deformation shifts the spectra in both perpendicular directions with respect to each other. If the flux expansion is large enough, as it typically is in the edge of a shaped tokamak equilibrium, specially when features like the Xpoint are present, then this variation of the perpendicular anisotropy along the magnetic field can cause non-convergence of simulations done within the resolution allowed by available computational cost [11]. This happens because the turbulence, which tends to be isotropic at small scales [7], [9], can not be resolved everywhere along the field lines. The solution to this problem is what is presented in the remaining part of this paper.

\section{COnformal TREATMEnt OF THE GeOMETRY}

The main idea behind the solution to the conformal deformation that is bound to all globally field aligned coordinate systems is to use those only for what they are best suited for, namely, the wave dynamics along the field lines. To treat the turbulent motion that occurs in the plane perpendicular to it, we devise another coordinate system which is built, not to be globally aligned with the magnetic field, but rather to keep the conformal ratio defined previously in Eq. (18) as constant as possible everywhere on the perpendicular mesh. The oneto-one and onto map between the two coordinates' systems is established through shifts in their angle-like coordinates, allowing a natural interaction between parallel and perpendicular motions in the most efficient possible way in terms of computational cost. The following subsections detail such a treatment, starting from the appropriate choice of Clebsch coordinates, then proving the map to a novel conformal coordinate system, whose construction is also presented.

\section{A. Symmetry coordinates}

The parallel wave dynamics, is to be solved on a Clebsch coordinate system on the grounds that they allow a computationally efficient coarser parallel mesh. The particular choice made here uses the so called symmetry flux coordinate system $(\Psi, \theta, \zeta=\phi)$, a standard choice in texts [12], sometimes also referred to as PEST coordinates [13]. $\psi$ is the toroidal component of the magnetic vector potential (also the poloidal flux $\Psi$ divided by $-2 \pi), \phi$ is the geometrical toroidal angle, and $\theta$ is a generalised poloidal angle coordinate, defined in a manner to yield a straight representation of the field lines in the angles (recall Sec. II-A).

The standard representation of the tokamak axisymmetric magnetic field is

$$
\mathbf{B}=I \nabla \phi+\nabla \psi \times \nabla \phi
$$

in cylindrical (polar) coordinates $(R, Z, \phi)$, where $I=I(\psi)$ is a measure of the poloidal current, and $\psi$ and $\phi$ are defined above. From this expression, the symmetry magnetic field contravariant components can be found by contraction with the respective coordinate gradients

$$
B^{\psi}=0 \quad B^{\phi}=\mathbf{B} \cdot \nabla \phi=\frac{I}{R^{2}} \equiv q B^{\theta} .
$$

The last equality defines what the generalised poloidal angle should be to ensure a constant field pitch, $q(\psi) \equiv B^{\phi} / B^{\theta}$, within a magnetic flux surface. The Jacobian for these coordinates follows directly, by noting that, from Eq. (20)

$$
B^{\theta}=\mathbf{B} \cdot \nabla \theta=\nabla \psi \times \nabla \phi \cdot \nabla \theta=\frac{1}{\sqrt{g}} .
$$

Invoking the general tensor transformation rules, one can reexpress the poloidal contravariant component of the magnetic field in terms of the poloidal parametric variable $\eta$, which can be identified for instance, with the geometrical poloidal angle

$$
\begin{aligned}
\mathbf{B} \cdot \nabla \theta & =\mathbf{B} \cdot\left(\frac{\partial \theta}{\partial \psi} \nabla \psi+\frac{\partial \theta}{\partial \eta} \nabla \eta+\frac{\partial \theta}{\partial \phi} \nabla \phi\right) \\
& =\left.\frac{\partial \theta}{\partial \eta}\right|_{\psi} \mathbf{B} \cdot \nabla \eta .
\end{aligned}
$$

The partial derivative with respect to $\eta$ is the only nonvanishing term due to (i) axisymmetry, and (ii) $B^{\psi} \equiv 0$. Substituting in Eq. (23) the expression for $B^{\theta}$ from Eq. (21) and integrating, one obtains

$$
\theta=\frac{I(\psi)}{q(\psi)} \int \frac{d \eta}{\mathbf{B} \cdot \nabla \eta} \frac{1}{R^{2}} .
$$

The normalisation requirement that the generalised poloidal symmetry angle coordinate has a period of $2 \pi$ defines the pitch parameter

$$
q(\psi)=\frac{I(\psi)}{2 \pi} \oint \frac{d \eta}{\mathbf{B} \cdot \nabla \eta} \frac{1}{R^{2}} .
$$


Lastly, one should note that the symmetry coordinates fulfil trivially the divergence-free property of the magnetic field

$$
\nabla \cdot \mathbf{B}=\frac{1}{\sqrt{g}} \frac{\partial}{\partial x^{\mu}}\left(\sqrt{g} B^{\mu}\right)=B^{\theta} \frac{\partial}{\partial \theta} \frac{B^{\theta}}{B^{\theta}}=0
$$

for which the expression for the Jacobian (22) has been used together with the axisymmetry property, and $x^{\mu} \in\{\psi, \theta, \phi\}$.

To avoid secular poloidal dependence of the anisotropy due to the magnetic shear, the shifted metric procedure is applied in its simplest form, as discussed in Sec. II-A. Hereby, a rigid poloidal shift $\phi_{k} / q(\psi)$ is applied, such that the final shifted field aligned symmetry coordinates become

$$
\begin{aligned}
& \chi=\psi \\
& \xi_{k}=\theta-\frac{\phi-\phi_{k}}{q} . \\
& \zeta=\phi
\end{aligned}
$$

\section{B. One-to one and onto map to conformal coordinates}

As part of the process of defining the conformal coordinate system, it is appropriate to start by analysing some general coordinate mapping properties. First, we postulate that both symmetry and conformal coordinates' systems use the geometrical toroidal angle $\phi$ for the toroidal coordinate, but they differ on the definition of the flux label coordinate, which is proportional to poloidal magnetic flux $\Psi=-2 \pi \psi$ in the former, and given by a not yet specified function of it $x(\psi)$ in the latter. Also the poloidal angle-like coordinate differ, and we shall keep the notation $\theta$ for the symmetry system, and use $\theta_{c}$ for the conformal one. Now, we note that the volume element is a scalar function and hence invariant under coordinate transformations. Hence, we have the following relation for the volume element expressed in these two coordinate systems, which is valid by hypothesis

$$
d V=|\sqrt{g} d \psi d \theta d \phi|=\left|\frac{\partial x}{\partial \psi} \sqrt{g_{c}} d \psi d \theta_{c} d \phi\right|=d V_{c}
$$

and which is equivalent to

$$
\sqrt{g} d \theta=f(\psi) \sqrt{g_{c}} d \theta_{c}
$$

where

$$
f(\psi) \equiv-\frac{\partial x}{\partial \psi} .
$$

The last expression establishes the transformation rule for the flux label coordinate on both coordinate systems. Next, we need to do the same for the generalised poloidal angles, and for that we first note the relation between the poloidal contravariant component of the magnetic field and the Jacobian

$$
\begin{aligned}
& B^{\theta}=\mathbf{B} \cdot \nabla \theta=\nabla \psi \times \nabla \phi \cdot \nabla \theta=(\sqrt{g})^{-1} \\
& B^{\theta_{c}}=\mathbf{B} \cdot \nabla \theta_{c}=\nabla \psi \times \nabla \phi \cdot \nabla \theta_{c}=\left(f(\psi) \sqrt{g_{c}}\right)^{-1},
\end{aligned}
$$

for symmetry and conformal coordinates, respectively, obtained from the standard expression for the tokamak axisymmetric magnetic field Eq. (20). Gathering Eqs. (28) and (30), we obtain the needed relation which provides the map between both poloidal angles, namely

$$
\left.\frac{d \theta_{c}}{B^{\theta_{c}}}\right|_{\psi}=\left.\frac{d \theta}{B^{\theta}}\right|_{\psi}
$$

with $\psi$ and $\phi$ held fixed, that is, along a poloidal contour of a given flux surface. From this transformation rule, all we need to do to construct $\theta_{c}$ from its symmetry counterpart $\theta$, is to specify what is the contravariant component $B^{\theta_{c}}$. This is done in the following section.

\section{Conformal coordinates}

Just as the symmetry poloidal angle-like coordinate was obtained from the expression for the corresponding contravariant component of the magnetic field, the same is done now for the conformal counterpart. In this case, it is not the condition that the field pitch must be a flux function which defines $B^{\theta_{c}}$, but rather we specify its expression directly. Based in the requirement that the (perpendicular) coordinate cells $\left(\psi, \theta_{c}\right)$ should not distort their shape as a field line is followed, we require the volume element to compensate the changes in the distance between flux surfaces, which vary inversely proportional to $|\nabla \psi|$. Hence, we want

$$
d V_{c}=\sqrt{g_{c}} d x d \theta_{c} d \phi \equiv \frac{R}{g^{x x}} d x d \theta_{c} d \phi,
$$

where the factor $R$ appears to balance the toroidicity effects. This defines the Jacobian as

$$
\frac{1}{\sqrt{g_{c}}} \equiv \frac{g^{x x}}{R}=\left(\frac{\partial x}{\partial \psi}\right)^{2} \frac{g^{\psi \psi}}{R}=f^{2}(\psi) \frac{g^{\psi \psi}}{R}
$$

where the chain rule was applied together with Eq. (29) to transform the contravariant radial metric component. From this equation together with Eq. (30), we find directly

$$
B^{\theta_{c}}=f(\psi) \frac{g^{\psi \psi}}{R} .
$$

An immediate consequence of this choice is that the field lines are no longer straight in this coordinate system, since the field pitch defined as $B^{\phi} / B_{c}^{\theta}=\alpha(\psi, \eta) \neq q(\psi)$ in these coordinates is no longer a flux function. Hence, as shown in Sec. II-A, it is not possible to construct one globally aligned coordinate with the magnetic field (no Clebsch representation of the magnetic field is possible). Nevertheless, we already showed in Eq. (31) that there is a one-to-one and onto map between $\theta_{c}$ and $\theta$. To find the expression for $\theta_{c}$ we substitute the expressions (21) and (34) into the field equation (31). Hence,

$$
\left.\frac{d \theta_{c}}{d \theta}\right|_{\psi}=\left.\frac{B^{\theta_{c}}}{B^{\theta}}\right|_{\psi}=\frac{f(\psi) q(\psi)}{I(\psi)} R g^{\psi \psi}
$$

and integrating over the symmetry poloidal angle $\theta$ leads to

$$
\theta_{c}=\frac{f(\psi) q(\psi)}{I(\psi)} \int d \theta R g^{\psi \psi} .
$$

The flux label function $f(\psi)$ is responsible for the $2 \pi$ normalisation of the poloidal conformal angle $\theta_{c}$, so it must be given by

$$
f(\psi)=\left(\frac{q(\psi)}{2 \pi I(\psi)} \oint d \theta R g^{\psi \psi}\right)^{-1}
$$

from which, together with Eq. (29), the conformal radial coordinate integral can be found, namely

$$
x(\psi)=-\int d \psi f(\psi) .
$$


It is important to note that while the map $\theta \leftrightarrow \theta_{c}$ exists on each flux surface, the radial derivative $\partial \theta_{c} / \partial \psi$ at constant $\theta$ does not vanish. This has consequences for the construction of metric elements as shown below.

Finally, it is easy to check that the conformal coordinate system also verifies the divergence free property of the background magnetic field

$$
\nabla \cdot \mathbf{B}=\frac{1}{\sqrt{g_{c}}} \frac{\partial}{\partial x^{\mu}}\left(\sqrt{g_{c}} B^{\mu}\right)=\frac{g^{x x}}{R} \frac{\partial}{\partial \theta_{c}}\left(\frac{1}{f(\psi)}\right)=0
$$

where the axisymmetry and the expressions for the conformal Jacobian Eq. (33) and magnetic field poloidal contravariant component Eq. (34) have all been used with $x^{\mu} \in\left\{x, \theta_{c}, \phi\right\}$.

For the sake of the notation, we re-label the conformal angle $\theta_{c}$ as $y$, simply to have poloidal plane described by $(x, y)$. We already know the expression for the Jacobian, given in Eq. (33). From this we can find also $g^{x x}$ since $g^{\psi \psi}$ is known from the symmetry coordinate system. To find the off-diagonal contravariant metric element $g^{x y}$, we invoke the tensor transformation rules and apply them with respect to the symmetry coordinates, $x^{\mu} \in\{\psi, \theta, \phi\}$, namely

$$
g^{x y}=\frac{\partial x}{\partial x^{\mu}} \frac{\partial y}{\partial x^{\nu}} g^{\mu \nu} .
$$

Using the definition Eq. (29) and the orthogonality relations $g^{\psi \phi}=g^{\theta \phi}=0$, we obtain

$$
g^{x y}=-f(\psi)\left(\frac{\partial \theta_{c}}{\partial \psi} g^{\psi \psi}+\frac{\partial \theta_{c}}{\partial \theta} g^{\theta \psi}\right) .
$$

With the expressions for $1 / \sqrt{g_{c}}, g^{x x}, g^{x y}$ and $g^{\phi \phi}=$ $1 / R^{2}$ at hand, we can now obtain the expression for the only remaining non-vanishing contravariant metric component, namely, $g^{y y}$. To do so, compare the definition Eq. (33) to the determinant of the Jacobian matrix to obtain

$$
\begin{aligned}
& \left(\frac{g^{x x}}{R}\right)^{2}=\left|\begin{array}{ccc}
g^{x x} & g^{x y} & 0 \\
g^{x y} & g^{y y} & 0 \\
0 & 0 & 1 / R^{2}
\end{array}\right|=\frac{g^{x x} g^{y y}-\left(g^{x y}\right)^{2}}{R^{2}} \\
& \Rightarrow g^{y y}=\frac{\left(g^{x x}\right)^{2}+\left(g^{x y}\right)^{2}}{g^{x x}}
\end{aligned}
$$

From the previous expression it has become clear why the particular choice for $B^{\theta_{c}}$ in Eq. (34) was made. The $1 / R$ factor cancels the one appearing in the RHS due to toroidicity, and the term $g^{\psi \psi}$, which leads to the $g^{x x}$ in the LHS, cancels the one appearing in the RHS, such that the conformal ratio defined in Eq. (18) reduces to

$$
R_{c} \equiv \frac{g^{y y}}{g^{x x}}=1+\left(\frac{g^{x y}}{g^{x x}}\right)^{2}=1+d^{2},
$$

where $d$ is the non orthogonal deformation defined in Eq. (15). For this system it is given by

$$
d \equiv \frac{g^{x y}}{g^{x x}} .
$$

This shows that the conformal coordinate system yields an unitary conformal ratio plus a correction due to its underlying nonorthogonality. Provided the latter is small, which means $d \lesssim 1$, the system behaves well, without the loss of physical representation associated with the strong magnetic field shaping typical of tokamaks. This shall be illustrated in following section, where this quantity is calculated for an actual tokamak MHD equilibrium.

A final consideration is in order, namely, regarding the drawbacks of using the symmetry/conformal geometry combination present herein. The numerical cost/complexity of implementing this treatment is similar to one of the standard field aligned treatment. The only price to pay is the loss of the axisymmetry being connected to a single coordinate, like happens in the flux-tube formulation (with poloidal as parallel). In this paper, the toroidal angle is the parallel coordinate, so that a purely toroidal derivative involves both of the angle coordinates. The consequence is that the truncation in the toroidal domain is disallowed, so that the entire flux surface must be carried in computations, leading to a higher cost. Nevertheless, the gains obtained from its relation to the conformal coordinates of which can handle MHD equilibria shaping in a much more robust way, fully justifies it. Moreover, current work is moving in the direction of global models anyway as computational resources make possible investigation of the interaction between the microturbulence and the dynamics at intermediate MHD scales. A last point refers to our plans for future generalisation of this treatment to 3-D MHD equilibria of either stellarators or tokamaks with magnetic ripple. For those, there is no toroidal axisymmetry, and a conformal treatment would represent no practical disadvantage, since the full flux surface must be carried anyway.

\section{RESUlTS}

In this section we apply the definitions of the coordinates introduced so far to an JET MHD equilibrium calculated with the CLISTE MHD solver [14]. We discuss the nonorthogonality deformation, measured by $d$, and the variation in the conformal ratio $R_{c}$. The plot in Fig. 3a shows the equidistant perpendicular grid obtained for equidistant field aligned symmetry coordinates Eq. (26). Just by looking at the grid, its already clear that the robustness of this coordinate system close to the separatrix breaks down. This is expected since at the X-point the straight field poloidal angle is not defined (recall the definition Eq. (24) and that there is no poloidal component of the magnetic field there). Moreover, its is also evident that the conformal deformation defined in Eq. (18) is also severe in that region. As the X-point proximity (bottom of the plots) causes flux expansion to occur with the resulting grid cell stretching in the radial direction, the property explained in Eq. (17) causes grid cell compression in the poloidal direction. The opposite happens on the outboard mid plane, where the flux compression due to the Shafranov shift causes the poloidal size of the grid cells to increase. This is quantified in the plot on the right side of the same figure. Note that, for clarity of the figure, the same color is used for values larger than 10 . We see that one does not need to be very close to the separatrix to experience conformal deformation of the grid, with $R_{c}$ varying between values smaller than unity to one order of magnitude higher along one poloidal turn on the flux surface. The middle plot (Fig. 3b) shows the nonorthogonality deformation $d$ which, except in close vicinity 


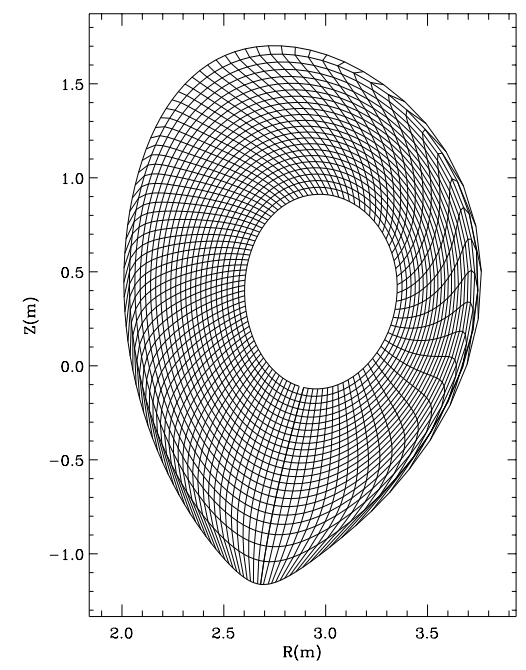

(a)

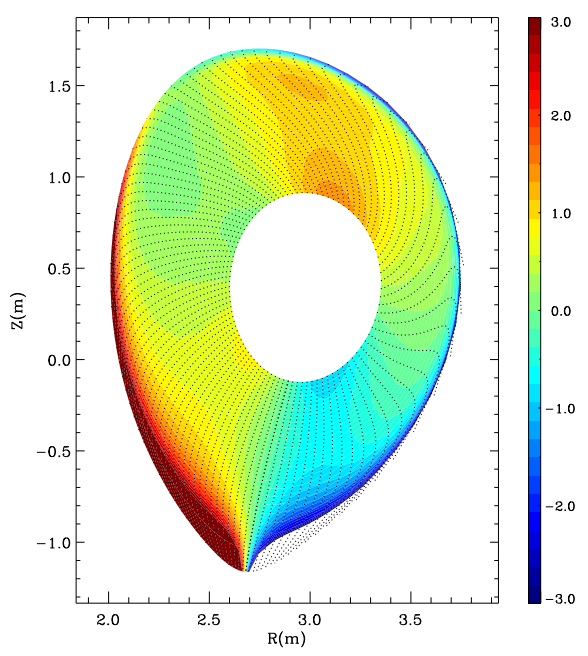

(b)

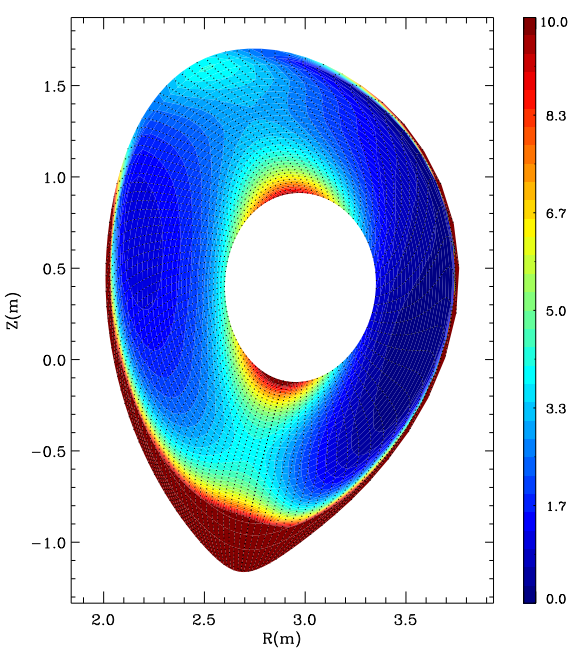

(c)

Fig. 3. Symmetry coordinates: (a) perpendicular (poloidal plane) equidistant grid; (b) non-orthogonal deformation as defined in Eq. (15). Note that the same color is used for values $\geq 3.0$ and that values $\leq-3.0$ are not represented, since this quantity diverges at the X-point; (c) conformal deformation $R_{c}$ as defined in Eq. (18). On the same grounds as before, no color distinction is made for values $\geq 10.0$.

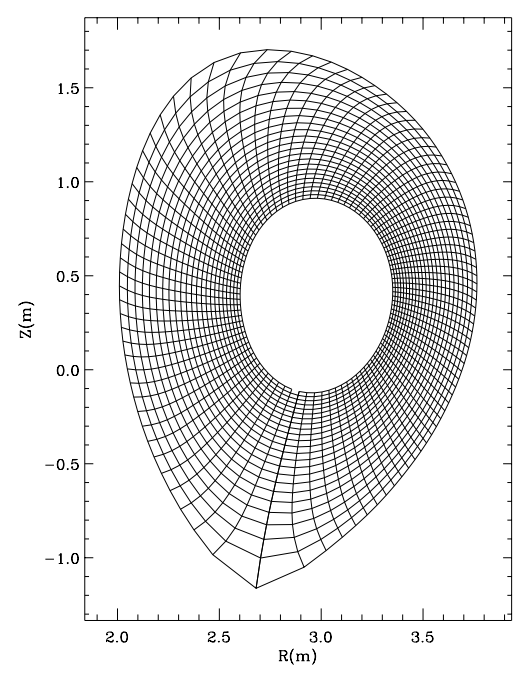

(a)

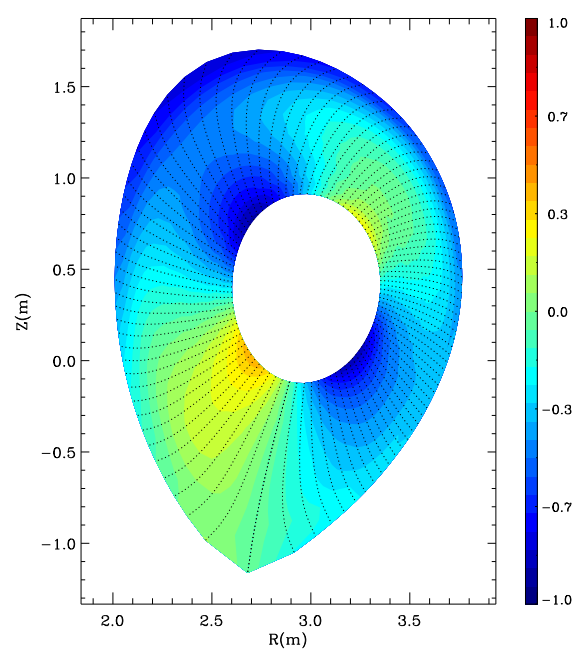

(b)

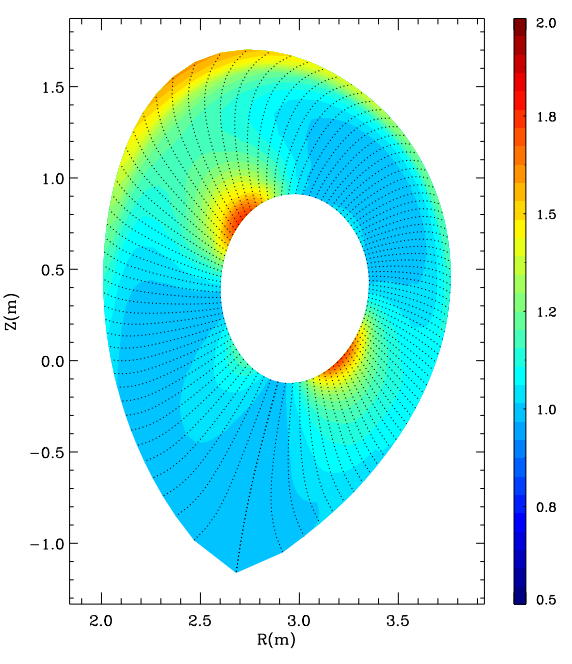

(c)

Fig. 4. Conformal coordinates: (a) perpendicular (poloidal plane) equidistant grid; (b) non-orthogonal deformation as defined in Eq. (15); (c) conformal deformation $R_{c}$ as defined in Eq. (18). Note the different color scales compared to Figs. $3 \mathrm{~b}$ and $3 \mathrm{c}$.

of the separatrix, behaves in a much sounder manner, with variations within the range \pm 1 . This is the result of the use of shifted metric procedure [6], without which values of large magnitude would be found. Fig. 4 shows the corresponding plots for the case of conformal coordinate system. In general is clear that these coordinates, as expected, are much more robust against the deformation issues which plague the standard field aligned coordinates, especially near the separatrix. From the equidistant grid [see Fig. 4a], we can see immediately the decrease in the radial grid spacing towards the center of the tokamak, which goes together with the natural decrease in poloidal spacing (see Appendix A). Note that, now, the nonorthogonal deformation, plotted in the middle, stays always within \pm 1 , even very close to the separatrix. More importantly, the conformal coordinates yield a conformal ratio which is everywhere within a factor of two of unity (note the different color scales between Figs. $3 b-3 c$ and $4 b-4 c$, respectively).

As discussed in Sec. II-C around Eq. (19), the isotropy of the turbulence at small scales [7], [9] is the physical reason to require grids which are close to isotropical. Since the minimum resolution common to both perpendicular coordinates is what matters, any resolution increase in one of them only is essentially wasted effort. The large values of grid anisotropy measured by the large variation of $R_{c}$ in symmetry coordinates indicates that this coordinate system has reduced practical applicability for the description of turbulent 
motions in the perpendicular plane in the edge of a shaped tokamak equilibrium, such as the one used here. Compared to the mostly isotropic conformal counterpart (which yields $R_{c} \approx 1$ everywhere), the symmetry coordinate system requires a resolution in the perpendicular coordinates which is higher by a factor of the order of its $R_{c}$ to solve the same problem. Especially near the separatrix this is severe enough $\left(R_{c}>10\right)$ to render the computation non-converged for reasonable (affordable) grid resolutions - e.g. of a typical ASDEX Upgrade edge simulation grid count of $64 \times 4094 \times 16$ in $\left(x, y_{k}, \zeta\right)$. Therefore, the symmetry coordinates should be used only for the parallel wave dynamics due to its global field alignment properties, while the perpendicular plane is to be handled by the conformal coordinates $(x, y)$. The latter can ensure the necessary degree of isotropy in the computational domain to resolve properly the turbulence at small scales at much less computational cost.

\section{SUMMARY AND OUTLOOK}

A novel geometrical treatment has been introduced. It uses the combination of two coordinate systems in such a way to make the most out of their strengths. They are related by a one-to-one and onto map on the poloidal angles, which makes the treatment consistent.

Conformal coordinates make possible a computational model for tokamak turbulence which is isotropic in the perpendicular plane at the grid spacing level, best representing its physical properties. An additional development presents itself: initial computations using this system with a multigrid method to solve equations of the form $\left(a-b \nabla_{\perp}^{2}\right) \phi=S$ has found that deformation impacts convergence only if the conformal ratio $R_{c}$ defined in Eq. (42) is more than a factor of two either way from unity. Typical shaped tokamak equilibria have been found to violate this only in small spatial regions, so that at the coarser grid levels the effect is averaged out. We have found that the conventional multigrid Poisson solvers using Cartesian geometry are adequate to the task, as the overall inhomogeneity factor $g^{x x} / R$ is sufficiently slowly varying so as not to cause problems on the finer grids. Hence, the use of conformal coordinates presents an ideal choice for global turbulence computation in realistic flux surface geometry.

Our future plans include performing gyrofluid simulations comparing flux tube and conformal geometries, both in simplified and realistic geometry, to make a quantitative assessment of the limits of both treatments. Although the latter is constructed to cope with stronger magnetic field shaping and, therefore, has a limit of applicability well beyond the former, it still depends on the usage of a Clebsch coordinate system to handle the parallel wave dynamics, which by definition is not defined at the $\mathrm{X}$-point. This implies that interpolation errors will become significant in the close vicinity to the $\mathrm{X}$ point, where the map between both coordinate systems has a singularity. Addressing this issue is left for future work. The conformal geometry described in this paper is being implemented in both gyrofluid and gyrokinetic models for turbulence computations [15]. Future generalisations to 3-D MHD equilibria are foreseen.

\section{APPENDIX}

\section{CYLINDER LIMIT CASE}

This section is devoted to the illustration of the differences between poloidal plane representations of the both symmetry (straight field line) and conformal coordinates, as defined in Secs. III-A and III-C, respectively. To do so, we are going to use a simplified circular problem within the large aspect ratio limit

$$
\epsilon \equiv \frac{r}{R} \ll 1 \quad R \rightarrow R_{0} \quad \psi=\psi(r),
$$

which reduces the problem to a cylindrical equilibrium by leaving out all toroidicity effects. In this case, since the field lines are by definition straight, it is easy see that the symmetry poloidal angle coincides with the geometrical one. To prove so mathematically, we need to calculate the geometrical poloidal contravariant component of the magnetic field by contracting Eq. (20) with the gradient of that coordinate

$$
B^{\eta}=\mathbf{B} \cdot \nabla \eta=\nabla \psi \times \nabla \eta \cdot \nabla \phi=-\frac{\psi_{r}^{\prime}}{r R}
$$

where $(r, \eta)$ are the geometrical polar coordinates in the poloidal plane, whose Jacobian is $(r R)^{-1}$ and $\psi_{r}^{\prime}=\partial \psi / \partial r$. The local magnetic field pitch for the polar coordinates is a flux function within the large aspect ratio limit

$$
\alpha=\frac{B^{\phi}}{B^{\eta}} \stackrel{\epsilon \ll 1}{\longrightarrow}-\frac{r I}{\psi_{r}^{\prime} R_{0}}=q(r) .
$$

where $B^{\phi}=I / R^{2}$. Substituting this into the symmetry poloidal angle Eq. (21) yields

$$
B^{\theta}=\frac{I}{q R^{2}}=-\frac{\psi_{r}^{\prime}}{r R}
$$

and hence Eq. (24) reduces to

$$
\theta=\int d \eta \frac{B^{\theta}}{B^{\eta}}=\int d \eta=\eta
$$

If we further approximate $\psi^{\prime}=1$, for the sake of simplicity of the illustration, we reach the result that the symmetry coordinates for our problem reduce to the geometrical cylindrical coordinates

$$
(r, \eta, \phi)
$$

Now we calculate the conformal coordinates for the same reduced problem. To find the poloidal conformal angle, we combine Eqs. (36) and (37)

$$
\theta_{c}=\frac{2 \pi \int d \theta R g^{\psi \psi}}{\oint d \theta R g^{\psi \psi}}
$$

and use

$$
R g^{\psi \psi} \stackrel{\epsilon \ll 1}{\longrightarrow} R_{0}\left[\psi_{r}^{\prime}(r)\right]^{2}
$$

to obtain directly

$$
\theta_{c}=\theta=\eta .
$$

For the remaining conformal coordinate $x$, all we need to do is to find the expression for $f(\psi)$ using Eqs. (37) and (45), namely,

$$
f(r)=-\frac{1}{r \psi_{r}^{\prime}}
$$




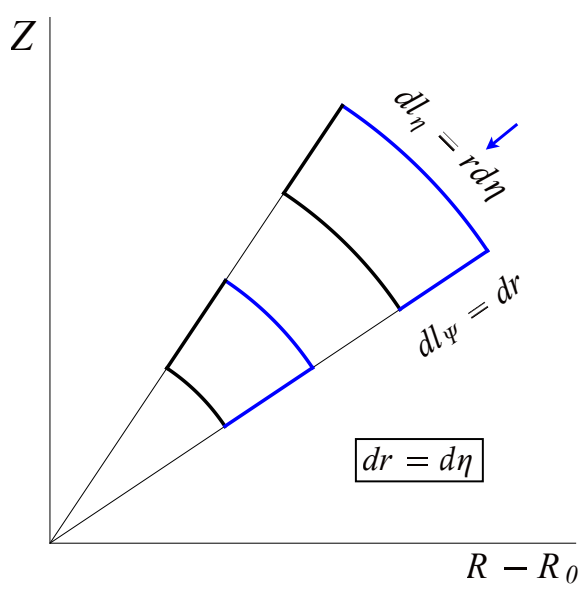

(a)

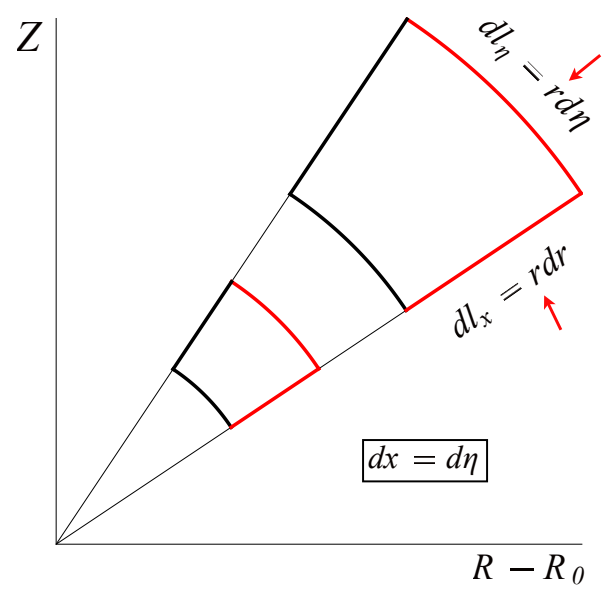

(b)

Fig. 5. (a) Illustration of the grid-cell deformation due to poloidal arc length radial dependence in symmetry (polar) coordinates. (b) Illustration of equivalent conformal grid, where the deformation problem has been corrected by including a radial dependence on the radial length.

and compare it to the definition of $x$ in Eq. (29) to obtain

$$
\frac{\partial x}{\partial r}=\frac{1}{r}
$$

Hence, within the $\epsilon \ll 1$ limit, the circular equilibrium conformal coordinate system is

$$
(\ln r, \eta, \phi) \text {. }
$$

The above analysis showed us that the only difference between both symmetry and conformal systems is the flux label coordinate. The point to note is that, the conformal one now leads to an extra factor of $r$ in the expression for the radial length element, as compared to the symmetry (polar) one

$$
d l_{x}=\sqrt{g^{x x}} d x=r d r \quad d l_{r}=\sqrt{g^{r r}} d r=d r .
$$

This factor of $r$ compensates the one in poloidal arc length

$$
d l_{\theta}=d l_{\theta_{c}}=d l_{\eta}=\sqrt{g^{\eta \eta}} d \eta=r d \eta .
$$

Hence, for symmetry coordinates, only one dimension (poloidal) expands with $r$, causing the grid cells to stretch in the poloidal direction as $r$ increases, as illustrated in Fig.5a. On the other hand, in the conformal coordinates, both dimensions expand such that the ratio of the grid cell sides length is kept constant. This is illustrated in Fig.5b.

\section{ACKNOWLEDGMENT}

The authors would like to thank Dr. Dirk Reiser for the motivating discussions on the subject of optimal geometrical descriptions of diverted tokamak MHD equilibria.

\section{REFERENCES}

[1] J. M. Greene and J. L. Johnson, "Stability criterium for arbitrary hydromagnetic equilibria," Physics of Fluids, vol. 5, no. 5, pp. 510 517, May 1962.

[2] R. L. Dewar and A. H. Glasser, "Ballooning mode spectrum in general toroidal systems," Phys. Fluids, vol. 26, no. 10, pp. 3038-3052, October 1983.
[3] S. C. Cowley, R. M. Kulsrud, and R. Sudan, "Considerations of iontemperature-gradient-driven turbulence," Phys. Fluids B, vol. 3, no. 10, pp. 2767-2782, October 1991.

[4] M. A. Beer, S. C. Cowley, and G. W. Hammett, "Field-aligned coordinates for nonlinear simulations of tokamak turbulence," Phys. Plasmas, vol. 2, no. 7, pp. 2687-2700, July 1995.

[5] B. Scott, "Global consistency for thin flux tube treatments of toroidal geometry," Phys. Plasmas, vol. 5, pp. 2334-2339, June 1998.

[6] - "Shifted metric procedure for flux tube treatments of toroidal geometry: Avoiding grid deformation," Phys. Plasmas, vol. 8, no. 2 , p. 447, February 2001.

[7] A. Kolmogorov, "The local structure of turbulence in an incompressible fluid at very large reynolds numbers," Dokl. Akad. Nauk SSSR, vol. 30, pp. 301-304, 1941.

[8] M. Wakatani and A. Hasegawa, "A collisional drift wave description of plasma edge turbulence," Phys. Fluids, vol. 27, pp. 611-618, March 1984.

[9] B. Scott, "The nonlinear drift wave instability and its role in tokamak edge turbulence," New J. Phys., vol. 4, pp. 52.1-52.30, 2002.

[10] B. D. Scott, "The mechanism of self-sutainment in collisional drift wave turbulence," Phys. Fluids B, vol. 4, pp. 2468-2494, August 1992.

[11] T. Ribeiro, "Turbulence studies in the scrape-off layer of tokamak plasmas by three-dimensional gyrofluid simulations," Ph.D. dissertation, Instituto Superior Técnico, Universidade Técnica de Lisboa, July 2005.

[12] R. D. Hazeltine and J. D. Meiss, Plasma Confinement, ser. Fronteirs in Physics. Addison-Wesley Publishing Company, 1992, vol. 86.

[13] R. Grimm, R. Dewar, and J. Manickam, "Ideal mhd stability calculations in axisymmetric toroidal coordinate systems," J. Comput. Phys., vol. 49, no. 1, pp. 94-117, 1983.

[14] P. J. McCarthy, P. Martin, and W. Schneider, "The CLISTE interpretative equilibrium code," Max-Planck-Institut für Plasmaphysik, Tech. Rep., May 1999.

[15] B. Scott, A. Kendl, and T. Ribeiro, "Nonlinear dynamics in the tokamak edge," Contrib. Plasma Phys., 2010, (accepted).

Tiago T. Ribeiro received the Ph.D. degree from the Universidade Técnica de Lisboa, Lisbon, Portugal, in 2005.

$\mathrm{He}$ entered the field of laboratory plasma research almost 10 years ago, doing experimental work on two major tokamaks: JET in UK and ASDEX Upgrade in Germany. He then continued into the field of theoretical plasma research. Since 2005, he has been with the Max-Planck-Institut fuer Plasmaphysik, in Germany, where he conducts his research work on the topic of turbulence in magnetised plasmas, in strong collaboration with the Instituto de Plasmas e Fusão Nuclear, Instituto Superior Técnico, Universidade Técnica de Lisboa. 
Bruce Scott is active in the field of magnetised plasma physics for over 25 years with over 60 published papers. His $\mathrm{PhD}$ from the University of Maryland dates from 1985. Since 1988 he is based at the Max-Planck-Institut fuer Plasmaphysik. 